\title{
Longitudinal stability of gel T1 MRI Phantoms for quality assurance of T1 mapping
}

\author{
Vassilis Vassiliou 1,3* Ee Ling Heng ${ }^{3,1}$, Jackie Donovan², Andreas Greiser ${ }^{4}$, Sonya V Babu-Narayan, 3,1 , \\ Michael A Gatzoulis ${ }^{3}$, David Firmin ${ }^{3,1}$, Dudley J Pennell ${ }^{1,3}$, Peter Gatehouse ${ }^{1,3}$, Sanjay K Prasad ${ }^{1,3}$ \\ From 18th Annual SCMR Scientific Sessions \\ Nice, France. 4-7 February 2015
}

\section{Background}

Quantification of interstitial (diffuse) myocardial fibrosis by T1 mapping may prove useful in a range of conditions. Both native T1 maps and extracellular volume fraction (ECV) are being increasingly used clinically and have been shown to be sensitive to a wide range of conditions and associate with worse prognosis. However, quality control by $\mathrm{T} 1$ phantoms is essential $[1,2]$ and their long-term stability has not been demonstrated. Phantoms with long term stability could assure the stability of methods applied to patients against scanner alternations and across multiple centers. We sought to investigate the long-term stability of phantoms prepared to model T1 and T2 of blood and myocardium over a period of 52 weeks.

\section{Methods}

$\mathrm{NiCl}_{2}$-agarose gel phantoms [3] were prepared in a standardised lab following a reproducible procedure to model $\mathrm{T} 1$ and $\mathrm{T} 2$ of blood and myocardium, native and post- contrast [4]. Phantoms were $60 \mathrm{ml}$ glass narrow-neck sealed thick-wall bottles, filled with minimal gaps from gel contraction whilst cooling. These were kept in a temperature controlled MRI room and imaged weekly for 52 consecutive weeks (Siemens, Avanto, 1.5T) using consistent coil and phantom arrangement with a 11-RR MOLLI prototype (Siemens WIP448B) with high resolution (for heart rate $75 \mathrm{bpm}$ ) and low resolution (for heart rate $100 \mathrm{bpm}$ ) versions, with pre-contrast and post-contrast variants (4) plus spin-echo T2. Image parameters were identical weekly except for automatic adjustments of flip-angle and reference frequency. T1 and T2 values were taken as mean values in each phantom in pixel-wise maps.

\section{Results}

Both high resolution and low resolution sequences had similar T1 means, standard deviation and coefficient of variation $(\mathrm{CoV})$ for blood and myocardium native and post-gadolinium (Table 1). CoV was higher for the longer T1 and T2 values. Specifically for the T1 values,

Table 1 showing the variability during a 52 week period of the Phantom T1 and T2 values and ECV using an 11-cycle 8-image prototype MOLLI. There were 2 phantoms for each T1 and T2 value prepared in standardised laboratory and the value used here represents the average of the two. The phantom ECV was calculated using a hematocrit of 0.43

\begin{tabular}{lccccccccc}
\hline \multicolumn{1}{c}{} & \multicolumn{1}{c}{ T1 } & & T2 \\
\hline & Mean (ms) & SD & CoV (\%) & Mean (ms) & SD & CoV (\%) & Mean (ms) & SD & CoV (\%) \\
\hline Native Myocardium & \multicolumn{1}{c}{ High Resolution (75bpm) } & Low resolution (100bpm) & Spin-echo protocol \\
\hline Native Blood & 972 & 12.6 & 1.3 & 972 & 13.6 & 1.4 & 56 & 1.3 & 2.2 \\
\hline Post Gd Myocardium & 1579 & 56.4 & 4.6 & 1550 & 61.9 & 4.0 & 234 & 8.0 & 3.4 \\
\hline Post Gd Blood & 498 & 5.1 & 1.0 & 497 & 13.5 & 1.4 & 48 & 0.9 & 1.9 \\
\hline ECV & 372 & 6.4 & 1.7 & 372 & 6.5 & 1.8 & 149 & 3.0 & 2.0 \\
\hline
\end{tabular}

${ }^{1}$ CMR, Royal Brompton Hospital, London, UK

Full list of author information is available at the end of the article 


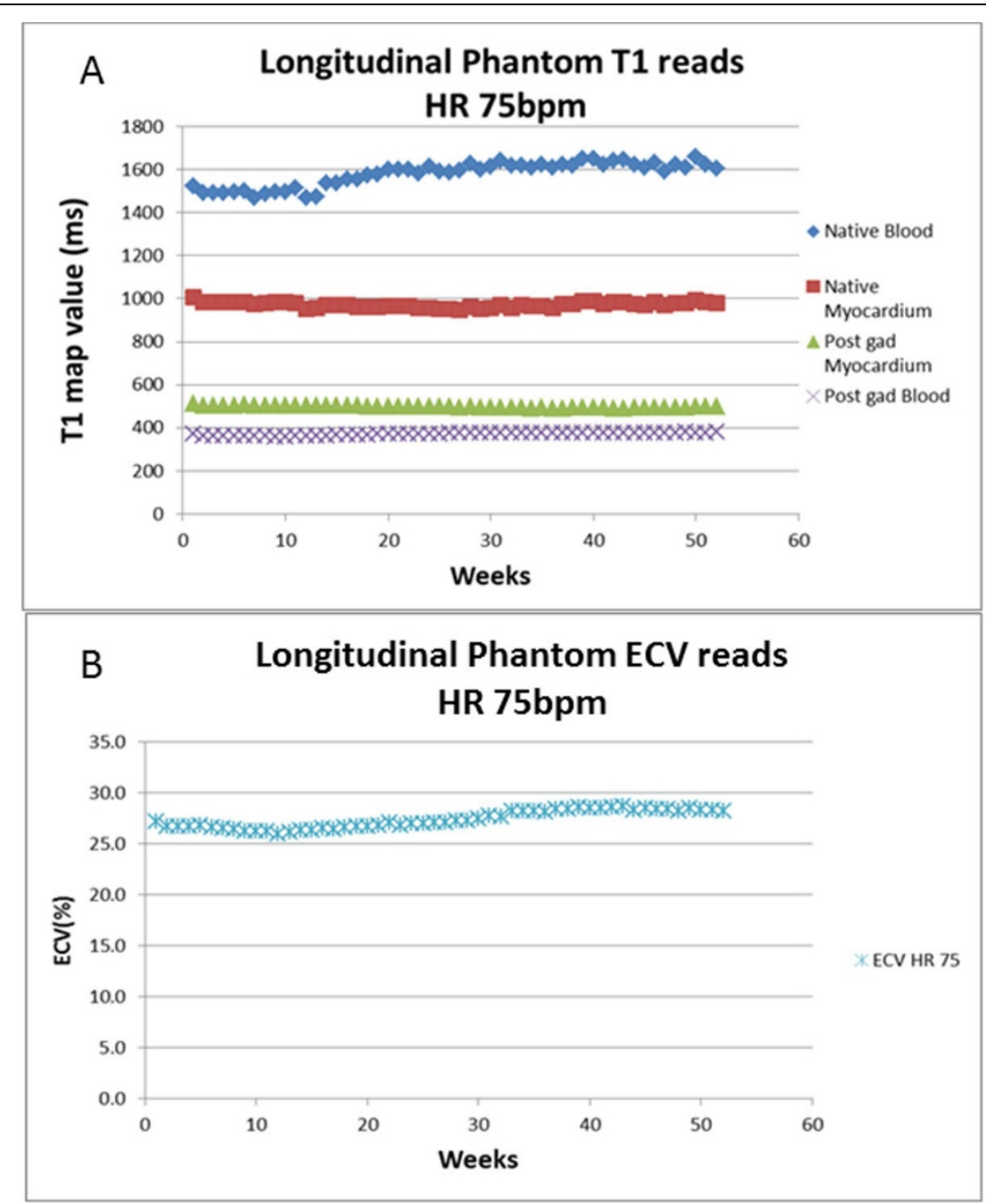

Figure 1 showing longitudinal trend for $\mathrm{T} 1$ maps and ECV. All $\mathrm{T} 1$ parameters remained relatively stable, with native myocardium remaining the most stable. This is particularly useful for $\mathrm{T} 1$ mapping sequences only utilizing native myocardial values. The ECV also remained relatively stable during these 52 weeks, suggesting that gel phantoms can be accurately used in the quality assurance of T1 mapping.

there was no significant drift seen in the shorter $\mathrm{T} 1$ values (native myocardium and post-gad blood and myocardium) but there was an increase in the longer native blood value of over one year in accordance to the following equation: T1 native blood $=3.25 \mathrm{x}$ no of weeks +1492 , suggesting an average increase of $169 \mathrm{~ms}$ over the year (Figure 1). Native blood $\mathrm{T} 1$ was the most stable parameter following: T1 native myocardium $=-0.01 \times$ no of weeks +972 suggesting a decrease of $1 \mathrm{~ms}$ over this period.

\section{Conclusions}

There was significant stability of the $\mathrm{NiCl}_{2}$-agarose gel phantoms over a one year period, justifying their routine use in clinical practice to support T1 mapping. Having determined stability, detection of parameters that could lead to phantom value inaccuracies (e.g. heart rate, flip angle and temperature variation) may require further investigation.

\section{Funding}

NIHR Cardiovascular Biomedical Research Unit of Royal Brompton \& Harefield NHS Foundation Trust and Imperial College London.

Authors' details

${ }^{1}$ CMR, Royal Brompton Hospital, London, UK. ²Department of Biochemistry, Royal Brompton Hospital, London, UK. ${ }^{3}$ National Heart and Lung Institute, Imperial College London, London, UK. ${ }^{4}$ Siemens, Germany, Germany.

Published: 3 February 2015

References

1. Messroghli: JMRI 2007. 
2. Gai: MRM 2013.

3. Kraft: MRM 1987.

4. Heng: JCMR 2014

doi:10.1186/1532-429X-17-S1-W28

Cite this article as: Vassiliou et al: Longitudinal stability of gel T1 MRI

Phantoms for quality assurance of T1 mapping. Journal of Cardiovascular Magnetic Resonance 2015 17(Suppl 1):W28.

Submit your next manuscript to BioMed Central and take full advantage of:

- Convenient online submission

- Thorough peer review

- No space constraints or color figure charges

- Immediate publication on acceptance

- Inclusion in PubMed, CAS, Scopus and Google Scholar

- Research which is freely available for redistribution

Submit your manuscript at 